Revolutionising the invisible orthodontic sector

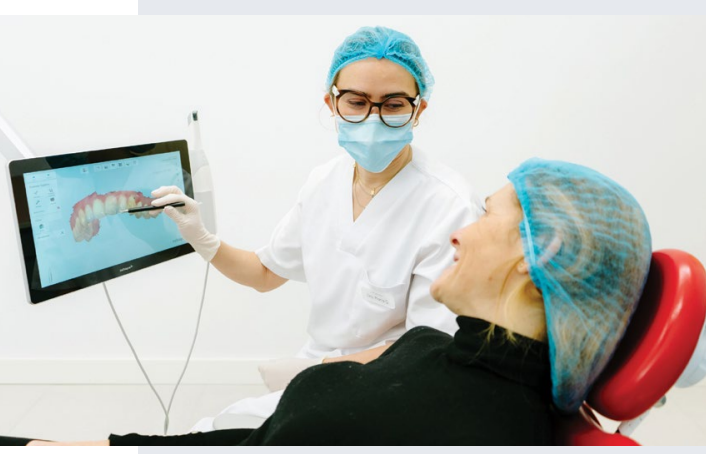

Impress is the leading chain of orthodontic clinics of a new generation that has revolutionised the invisible orthodontic sector with the best team of professionals specialised in making people smile and the latest technology applied to the diagnosis, treatment and follow-up of all cases.

Impress is the largest chain of ortho clinics with fully digital processes, including its own clinic management system and remote treatment-monitoring platform. Impress currently has two clinics in the UK: London and Manchester, with flagships to be opened in London and Manchester in a matter of weeks.

Born in 2019 with the aim of caring for and improving the smile of patients without losing sight of their oral health, Impress is the only company specialised in invisible orthodontics that has an internal team of professionals that works with the latest medical technology. In fact, Impress allocates all their investment to the latest technology in orthodontics so they can offer the most advanced treatment and service; the 3D scan, video results of the treatment and digital monitoring are just some of the innovations in the sector.

Impress orthodontists, who specialise in oral health, assess each case individually to achieve the desired result. For them, there is no better reward than making a client smile. Treatments can vary from patient to patient as every smile is unique, although $80 \%$ of cases are complete within six months or less.

In less than two years, Impress has managed to position itself as the European leader in the orthodontic sector, with no signs of slowing down.

For more information, visit www. smile2impress.com.

\title{
The dental camera for your practice or laboratory
}

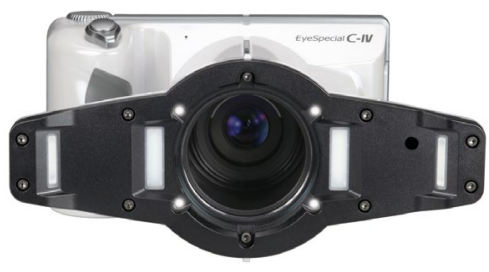

The Shofu EyeSpecial dedicated dental camera has everything your dental practice or laboratory really needs. It possesses smart special features, is easy to operate, reproducibly takes excellent images, and its use can be delegated to your assistants without any lengthy training.

The Shofu EyeSpecial has built-in photographic expertise. The camera relieves users of their worries about ring flash, aperture, depth of field etc and has everything needed to easily take informative patient images. Thanks to its smart integrated features, the EyeSpecial reliably produces excellent photos, without any time-consuming alignment of flashes or other settings and adjustments.

The EyeSpecial supports modern hygiene practices in your operatory. The completely smooth camera body can be quickly and thoroughly disinfected between patients, and menu navigation on the touch panel works properly even when wearing disposable gloves.

This ultralight high-performance camera does not require any heavy accessories. It can easily be held with one hand, freeing the other to hold a cheek retractor or a mirror, and the integrated flash system provides the optimal light, irrespective of the ambient light conditions.

Just like its predecessor, the new EyeSpecial C-IV has special shooting modes for ease of use and quicker results. Thanks to the autofocus feature, you can concentrate on subject and shutter or delegate photo documentations to your assistants. The camera now offers a video feature for case documentations in 'motion pictures' and an optional cross polariser, allowing you to look inside a tooth without any light reflections.

Download the product brochure at https://www.shofu.de/en/produkt/ eyespecial-c4-uk/. Do you wish to see a demonstration and take advantage of an attractive offer? Your Shofu representative will be happy to assist you. Contact the Shofu UK office on 01732783580 or sales@ shofu.co.uk, www.shofu.co.uk.

\section{More than just a surgical motor}

The Surgic Pro2 has been designed to enhance the performance of the popular Surgic Pro with a range of new specifications to ensure greater comfort, efficiency and safety during implant treatment and oral surgery.

It is easier to use, with a lighter, more compact motor; it has an improved LCD display - a larger, back-lit, high contrast LCD panel, adjustable to ten different brightness levels; and it has a long-lasting colour LED light source.

A large part of the success of the Surgic Pro has been as a result of the unique NSK Advanced Handpiece Calibration (AHC) function. This guarantees more accurate speed and torque control, and the new Surgic Pro2 takes this further still. The accuracy of the torque value is dependent on the state of the handpiece (bearings and gear abrasion) which the Surgic Pro2 calibration now takes into account to display more accurate values. AHC corrects the differences in the handpiece's condition by minimising the

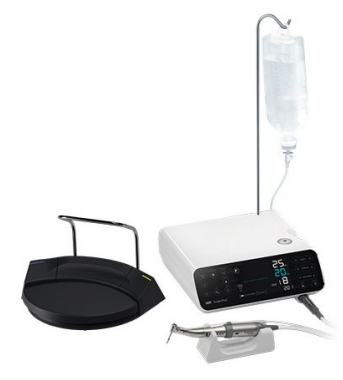

discrepancy between the set torque value and the actual output which helps to improve overall treatment outcomes.

The Surgic Pro2 can also be used as the central interface for other primary surgical equipment. It links by Bluetooth to an iPad, the NSK VarioSurg3 Piezo surgical unit, the NSK Osseo100+ implant stability measurement device and a wireless foot control. Using the free NSK app means all patient and treatment-related data generated during procedures can be visualised and saved to an external device.

To find out more visit www.uk.nsk-dental. com/products/surgical/surgical-surgicpro2/. 